\title{
CHARACTERIZATION OF DISTRIBUTIONS BY EQUALITIES OF TWO GENERALIZED OR DUAL GENERALIZED ORDER STATISTICS
}

\author{
Magdy E. El-Adll \\ Mathematics Department, Faculty of Science, Helwan University, Ain Helwan, Cairo, Egypt.
}

Received 23/1/2018 $\quad$ Revised Accepted 31/7/2018

\section{Abstract}

In this paper, characterization of probability distributions by equalities of two different generalized order statistics (gos) or dual generalized order statistics (dgos) is considered. It is proved that, if two different gos or dgos via the same distribution function (df) $F$ are equal, then $F$ has at most two growth points.

Keywords: generalized order statistics; dual generalized order statistics; characterization of distributions.

MSC2010: 62E10; 62E15; 62G30.

\section{Introduction}

Generalized order statistics have been introduced by Kamps 1 as an integrated approach of varied models of ascending ordered random variables (rv's) including, ordinary order statistics, sequential order statistics, progressive type II censored order statistics, record values, and Pfeifer's records. The gos $Y\left(i, n, \tilde{m}_{n}, k\right), i=1,2, \ldots, n$ with $n \geqslant 2$, based on a df $F$ are defined via their joint probability density function (jpdf),

$$
f_{1,2, \ldots, n}\left(y_{1}, y_{2}, \ldots, y_{n}\right)=k\left(\prod_{j=1}^{n-1} \gamma_{j}\right)\left(\prod_{j=1}^{n-1}\left[1-F\left(y_{j}\right)\right]^{m_{j}} f\left(y_{j}\right)\right)\left[1-F\left(y_{n}\right)\right]^{k-1} f\left(y_{n}\right),
$$

on the cone $F^{-1}(0+)<y_{1} \leqslant \cdots \leqslant y_{n}<F^{-1}(1)$, with parameters $\gamma_{r}=\gamma_{r}\left(n, \tilde{m}_{n}, k\right)=k+n-r+M_{r}>0, r=1,2, \ldots, n$, where $M_{r}=\sum_{j=r}^{n-1} m_{j} ; M_{n}:=0$ and $\tilde{m}_{n}=\left(m_{1}, m_{2}, \ldots, m_{n-1}\right) \in \mathbb{R}^{n-1}$. Specific choices of the model parameters $\gamma_{i}$ lead to particular models, e.g., ordinary order statistics (oos), sequential order statistics (sos), progressive type II censored order statistics, standard, $k$ th and Pfeifer's record values. Alternatively, in $[2]$ gos have been defined as the product of independent power function distributed rv's, $B_{j}, 1 \leqslant j \leqslant n$. Namely,

$$
Y\left(r, n, \tilde{m}_{n}, k\right) \stackrel{d}{=} F^{-1}\left(1-\prod_{j=1}^{r} B_{j}\right) \stackrel{d}{=} F^{-1}\left(1-\prod_{j=1}^{r} U^{\frac{1}{\gamma_{j}\left(n, \tilde{m}_{n}, k\right)}}\right), r=1,2, \ldots, n,
$$

where $U$ is a standard uniform rv and $X \stackrel{d}{=} Y$ stands for $X$ and $Y$ have the same df. Consequently, $Y\left(1, n, \tilde{m}_{n}, k\right) \leqslant$ $Y\left(2, n, \tilde{m}_{n}, k\right) \leqslant \ldots \leqslant Y\left(n, n, \tilde{m}_{n}, k\right)$ holds almost surely.

The $m$-generalized order statistics $\left(m\right.$-gos) is a wide subclass of gos corresponding to the special choice $m_{1}=m_{2}=\ldots=$ $m_{n-1}=m$ i.e. $\gamma_{r}\left(n, \tilde{m}_{n}, k\right)=\gamma_{r}(n, m, k)=k+(n-r)(m+1)$. The $m$-gos model includes, oos $\left(k=1, m=0\right.$ i.e. $\gamma_{r}(n, m, k)=$ $n-r+1)$ and $k$ th upper record values $\left(k \in \mathbb{N}, m=-1\right.$ i.e. $\left.\gamma_{r}(n, m, k)=k\right)$, with $r=1,2, \ldots, n$, as special cases. The reader is referred to, e.g., [1], 2], [3], [4], and [5 for more details. The marginal df of the $r$ th $m$-gos, $Y(r, n, m, k)$, is obtained in 1. Namely,

$$
F_{Y(r, n, m, k)}(y)=1-C_{r}(n, m, k) \bar{F}^{\gamma_{r}(n, m, k)}(y) \sum_{j=0}^{r-1}\left(\Gamma(j+1) C_{r-j}(n, m, k)\right)^{-1} g_{m}^{j}(F(y)),
$$


where,

$$
\begin{gathered}
g(y)= \begin{cases}\frac{1}{m+1}\left[1-(1-y)^{m+1}\right], & m \neq-1 ; \\
-\log (1-y), & m=-1,\end{cases} \\
C_{r}(n, m, k):=\prod_{i=1}^{r} \gamma_{i}(n, m, k), \quad r=1,2, \ldots, n, \quad \text { and } \quad \bar{F}=1-F .
\end{gathered}
$$

Clearly, the function $G_{m}(y):=(m+1) g_{m}(F(y))=1-\bar{F}^{m+1}(y)$, is a df, for $m \neq-1$.

A characterization in statistics is a specific distributional property of a statistic that uniquely identify related parametric family of distributions. Classical results in characterizations can be found in [6], 7] and 8 . For a comprehensive survey of characterizations on the basis of functions of order statistics, see Gather et al [9]. Since gos have been introduced in 1], characterization of probability distributions based on ordered rv's receives an increasing attention by several authors including, 10], 11] and [12, among others.

In this paper, the results of 13 for ordinary order statistics, are extended to gos and dgos models.

\section{Characterization of Distribution Based on gos}

In this section, all possible situations when two different gos have the same distribution function are discussed. In other words, if $Y\left(i, n_{1}, \tilde{m}_{n_{1}}, k\right), i=1,2, \ldots, n_{1}, Y\left(j, n_{2}, \tilde{m}_{n_{2}}, k\right), j=1,2, \ldots, n_{2}$ are gos based on the same df $F$, such that $Y\left(r, n_{2}, \tilde{m}_{n_{2}}, k\right) \stackrel{d}{=} Y\left(s, n_{1}, \tilde{m}_{n_{1}}, k\right)$, that is,

$$
F_{Y\left(r, n_{2}, \tilde{m}_{n_{2}}, k\right)}(y)=F_{Y\left(s, n_{1}, \tilde{m}_{n_{1}}, k\right)}(y), \quad \forall y \in \mathbb{R},
$$

what are the conclusions that can be obtained concerning $F$ ? Before answering this question, we define the following set of integers,

$$
\mathcal{L}=\left\{\left(n_{1}, n_{2}, r, s\right): n_{2}-n_{1}>r-s>0\right\}
$$

and let $\mathcal{L}^{C}$, denote the complement set of $\mathcal{L}$. The main results in this section are formulated in theorems 2.1 and 2.2 .

Theorem 2.1. Suppose that $Y\left(i, n_{1}, \tilde{m}_{n_{1}}, k\right), i=1,2, \ldots, n_{1}, Y\left(j, n_{2}, \tilde{m}_{n_{2}}, k\right), j=1,2, \ldots, n_{2}$ are gos based on the same $d f$ $F$, where $\left(n_{1}, n_{2}, r, s\right) \in \mathcal{L}^{C}$. Suppose also that 2.1) holds and the following condition,

$$
m_{r+1} \geqslant m_{r+2} \geqslant \cdots \geqslant m_{n_{2}-1},
$$

is satisfied. Then $F$ is degenerate $d f$.

The proof of the theorem is split into three lemmas. The first lemma includes some ordered relations which will be needed in the sequel, while the second lemma is formulated in the general case and include three cases in which the df $F$ is degenerate.

Lemma 2.1. Let $n, r, t$ be positive integers and let $m_{j} \geqslant-1$ be real numbers for all $j=1,2, \ldots, n+t-1$, such that $m_{r+1} \geqslant m_{r+2} \geqslant \cdots \geqslant m_{n+t-1}$. Then,

$$
Y\left(r, n, \tilde{m}_{n}, k\right) \leqslant Y\left(r+t, n+t, \tilde{m}_{n+t}, k\right), \quad \text { with probability one. }
$$

Proof. Clearly, $\gamma_{i}\left(n, \tilde{m}_{n}, k\right)-\gamma_{i+1}\left(n, \tilde{m}_{n}, k\right)=m_{i}+1 \geqslant 0, \forall i=1,2, \ldots, n$. Therefore, the ordered relation

$$
\gamma_{1}\left(n, \tilde{m}_{n}, k\right) \geqslant \gamma_{2}\left(n, \tilde{m}_{n}, k\right) \geqslant \cdots \geqslant \gamma_{n}\left(n, \tilde{m}_{n}, k\right)=k
$$

holds true. Similarly, by noting that $\gamma_{i}\left(n+t, \tilde{m}_{n+t}, k\right)-\gamma_{i}\left(n, \tilde{m}_{n}, k\right)=\sum_{j=n}^{n+t-1}\left(m_{j}+1\right) \geqslant 0$, I have

$$
\gamma_{i}\left(n+t, \tilde{m}_{n+t}, k\right) \geqslant \gamma_{i}\left(n, \tilde{m}_{n}, k\right), \quad \forall i=1,2, \ldots, n .
$$

Set $V_{s}(\ell)=\prod_{j=1}^{s} U^{\frac{1}{\gamma_{j}\left(\ell, \tilde{m}_{\ell}, k\right)}}$, where $U$ is a standard uniform random variable. From the assumptions of the lemma, I get

$$
\gamma_{j+t}\left(n+t, \tilde{m}_{n+t}, k\right)=k+n-j+\sum_{i=j+t}^{n+t-1} m_{i} \leqslant k+n-j+\sum_{i=j}^{n-1} m_{i}=\gamma_{j}\left(n, \tilde{m}_{n}, k\right) .
$$


Hence, we get

$$
\begin{aligned}
V_{r}(n)=\prod_{j=1}^{r} U^{\frac{1}{\gamma_{j}\left(n, \tilde{m}_{n}, k\right)}} & \geqslant \prod_{j=1}^{r} U^{\frac{1}{\gamma_{j+t}\left(n+t, \tilde{m}_{n+t}, k\right)}} \\
& =\prod_{i=t+1}^{r+t} U^{\frac{1}{\gamma_{i}\left(n+t, \tilde{m}_{n+t}, k\right)}} \geqslant \prod_{j=1}^{r+t} U^{\frac{1}{\gamma_{j}\left(n+t, \tilde{m}_{n+t}, k\right)}}=V_{r+t}(n+t) .
\end{aligned}
$$

Thereby,

$$
V_{r+t}(n+t) \leqslant V_{r}(n) \text {. }
$$

By the right continuity of $F$, according to the results of 14 , p.3 or 15$],$ p.15, $F\left(F^{-1}(u)\right) \geqslant u$ and $F^{-1}(F(x)) \leqslant x$. Therefore, $u \leqslant F(z)$ iff $F^{-1}(u) \leqslant z$. Hence by $(1.1)$ together with $(2.4)$, the lemma is proved.

Lemma 2.2. Let (2.1) be satisfied with $\left(n_{1}, n_{2}, r, s\right) \in \mathcal{L}^{C}$. Then $F$ is degenerate df, if one of the following three conditions holds.

$$
\begin{array}{ll}
\mathcal{C}_{1}: & 1 \leqslant r<s \leqslant n_{1}=n_{2}=n . \\
\mathcal{C}_{2}: & 1 \leqslant r=s \leqslant n_{1}<n_{2} . \\
\mathcal{C}_{3}: & 1 \leqslant r<s \leqslant n_{1}<n_{2} .
\end{array}
$$

Proof. For the first case, if $\mathcal{C}_{1}$ is satisfied, 2.1) reads $P\left(Y\left(r, n, \tilde{m}_{n}, k\right) \leqslant y\right)=P\left(Y\left(s, n, \tilde{m}_{n}, k\right) \leqslant y\right)$ with $r<s$, and $y \in \mathbb{R}$, which by 1.1 can be written as

$$
P\left(F^{-1}\left(1-V_{r}(n)\right) \leqslant y\right)=P\left(F^{-1}\left(1-V_{s}(n)\right) \leqslant y\right) .
$$

Hence 2.5 implies

$$
P\left(V_{r}(n) \geqslant \bar{F}(y)\right)=P\left(V_{s}(n) \geqslant \bar{F}(y)\right) .
$$

Since $r<s$ and $U \sim U(0,1), V_{r}(n)>V_{s}(n)$ with probability one. Thus by 2.6 I get

$$
0=P\left(V_{r}(n) \geqslant \bar{F}(y)\right)-P\left(V_{s}(n) \geqslant \bar{F}(y)\right)=P\left(V_{s}(n) \leqslant \bar{F}(y), V_{r}(n)>\bar{F}(y)\right), \quad \forall y,
$$

which holds only when $\bar{F}(y)=0$ or 1 . To prove the second case, first note that under condition $\mathcal{C}_{2}$, 1.1 and $(2.1)$ yield

$$
P\left(V_{r}\left(n_{1}\right) \geqslant \bar{F}(y)\right)=P\left(V_{r}\left(n_{2}\right) \geqslant \bar{F}(y)\right), \quad \forall y, \quad 1 \leqslant r \leqslant n_{1}<n_{2} .
$$

In view of 2.3$), V_{r}\left(n_{1}\right)<V_{r}\left(n_{2}\right)$ almost sure. Consequently,

$$
P\left(V_{r}\left(n_{1}\right) \leqslant \bar{F}(y), V_{r}\left(n_{2}\right)>\bar{F}(y)\right)=0 \quad \forall y,
$$

which corresponds to degenerate distribution. If condition $\mathcal{C}_{3}$ holds, 1.1 implies

$$
P\left(V_{r}\left(n_{2}\right) \geqslant \bar{F}(y)\right)=P\left(V_{s}\left(n_{1}\right) \geqslant \bar{F}(y)\right), \quad \forall y .
$$

Again, an application of 2.3 yields, $V_{r}\left(n_{2}\right)>V_{s}\left(n_{1}\right)$ with probability one. Thus

$$
P\left(V_{s}\left(n_{1}\right) \leqslant \bar{F}(y), V_{r}\left(n_{2}\right)>\bar{F}(y)\right)=0, \quad \forall y .
$$

Hence $F$ is degenerate df. The lemma is thus established.

Lemma 2.3. Under the same conditions of Theorem 2.1, if relation 2.1] is satisfied for $1 \leqslant n_{1}<n_{2}$ with $n_{2}-r \leqslant n_{1}-s$. Then $F$ is degenerate $d f$.

Proof. According to Lemma 2.1, the following ordered relation holds true

$$
Y\left(r, n_{2}, \tilde{m}_{n_{2}}, k\right) \geqslant Y\left(r+n_{1}-n_{2}, n_{1}, \tilde{m}_{n_{1}}, k\right) \geqslant Y\left(s, n_{1}, \tilde{m}_{n_{1}}, k\right)=Y\left(r, n_{2}, \tilde{m}_{n_{2}}, k\right),
$$

which implies $Y\left(r+n_{1}-n_{2}, n_{1}, \tilde{m}_{n_{1}}, k\right) \stackrel{d}{=} Y\left(s, n_{1}, \tilde{m}_{n_{1}}, k\right)$. Hence by the first case of Lemma 2.3 . $F$ must be degenerate df. 
In the previous four cases, it has been shown that $F$ is degenerate $\mathrm{df}$. The last case is given in the following theorem.

Theorem 2.2. Assume that $\left(n_{1}, n_{2}, r, s\right) \in \mathcal{L}$ and $m_{1}, \ldots, m_{n_{2}-1}$ are real numbers such that $m_{1}=m_{2}=\ldots=m_{r-1}=m$ and $\sum_{j=n_{1}}^{n_{2}-1} m_{j}>(r-s) m$, with $m_{j}>-1$. If $Y\left(r, n_{2}, \tilde{m}_{n_{2}}, k\right) \stackrel{d}{=} Y\left(s, n_{1}, \tilde{m}_{n_{1}}, k\right)$, then $F$ has exactly two growth points.

In order to prove the theorem, a simple representation for the marginal df of the $r$ th gos, $Y(r, n, \tilde{m}, k)$, with less restrictive conditions than $m$-gos, is given in the following lemma.

Lemma 2.4. Let $m_{1}=m_{2}=\cdots=m_{r-1}=m>-1$ and $\bar{m}_{r, n}=\frac{1}{n-r} \sum_{j=r}^{n-1} m_{j}>-1$, for $r \in\{2, \ldots, n\}$, be the mean of the numbers $m_{r}, \ldots, m_{n-1}$. Then

$$
F_{Y\left(r, n, \tilde{m}_{n}, k\right)}(y)=1-\sum_{j=0}^{r-1} \frac{\Gamma\left(N_{r, n}-r m_{r, n}^{*}\right)}{\Gamma(j+1) \Gamma\left(N_{r, n}-r m_{r, n}^{*}-j\right)} G_{m}^{j}(y) \bar{G}_{m}^{N_{r, n}-r m_{r, n}^{*}-j-1}(y),
$$

where

$$
N_{r, n}=\frac{k}{m+1}+\left(\frac{\bar{m}_{r, n}+1}{m+1}\right) n \quad \text { and } \quad m_{r, n}^{*}=\frac{m-\bar{m}_{r, n}}{m+1} .
$$

For $m_{1}=m_{2}=\cdots=m_{n-1}=m>-1$, we have $\bar{m}_{r, n}=m$ and $m_{r, n}^{*}=0$, which leads to

$$
F_{Y\left(r, n, m_{n}, k\right)}(y)=1-\sum_{j=0}^{r-1} \frac{\Gamma(N)}{\Gamma(j+1) \Gamma(N-j)} G_{m}^{j}(y) \bar{G}_{m}^{N-j-1}(y)
$$

where $N=n+\frac{k}{m+1}$.

Proof. According to lemma 1.1 of [16], I get

$$
F_{Y\left(r, n, \tilde{m}_{n}, k\right)}(y)=\mathrm{I}_{G_{m}(y)}\left(r, N_{r, n}-R_{r, n}\right),
$$

where

$$
\mathrm{I}_{z}(a, b)=\frac{1}{B(a, b)} \int_{0}^{z} t^{a-1}(1-t)^{b-1} d t
$$

denote the incomplete beta function,

$$
N_{r, n}=\frac{k}{m+1}+\left(\frac{\bar{m}_{r, n}+1}{m+1}\right) n \quad \text { and } \quad R_{r, n}=\left(\frac{\bar{m}_{r, n}+1}{m+1}\right) r .
$$

If $\alpha$ is an integer, it can be proved that

$$
\begin{aligned}
\mathrm{I}_{z}(\alpha, \beta) & =1-\sum_{j=0}^{\alpha-1} \frac{\Gamma(\alpha+\beta)}{\Gamma(\alpha-j) \Gamma(\beta+j-1)} z^{\alpha-1-j}(1-z)^{\beta+j} \\
& =1-\sum_{j=0}^{\alpha-1} \frac{\Gamma(\alpha+\beta)}{\Gamma(j+1) \Gamma(\beta+\alpha-j)} z^{j}(1-z)^{\alpha+\beta-1-j} .
\end{aligned}
$$

The lemma immediately follows by putting $\alpha=r$ and $\beta=N_{r, n}-R_{r, n}$ in 2.12).

Proof. of Theorem 2.2. Let $D(y)=F_{Y\left(r, n_{2}, \tilde{m}_{n_{2}}, k\right)}(y)-F_{Y\left(s, n_{1}, \tilde{m}_{n_{1}}, k\right)}(y)$. By the assumptions of the theorem with the same notations of Lemma 2.4 we have

$$
D(y)=\mathrm{I}_{G_{m}(y)}\left(r, N_{r, n_{2}}-R_{r, n_{2}}\right)-\mathrm{I}_{G_{m}(y)}\left(s, N_{s, n_{1}}-R_{s, n_{1}}\right)=0,
$$

which by setting $u=F(y)$ become

$$
\begin{aligned}
D(u)= & \frac{1}{\beta\left(r, N_{r, n_{2}}-R_{r, n_{2}}\right)} \int_{0}^{G_{m}(u)} t^{r-1}(1-t)^{N_{r, n_{2}}-R_{r, n_{2}}-1} d t \\
& \quad-\frac{1}{\beta\left(s, N_{s, n_{1}}-R_{s, n_{1}}\right)} \int_{0}^{G_{m}(u)} t^{s-1}(1-t)^{N_{s, n_{1}}-R_{s, n_{1}}-1} d t .
\end{aligned}
$$


Since $D(0)=D(1)=0$, it is sufficient to study the sign of the derivative $D^{\prime}(u)$ in the interval $(0,1)$, to determine the number of roots of $D(u)=0$ in the interval $[0,1]$. The derivative $D^{\prime}(u)$ may be written in the form

$$
D^{\prime}(u)=\frac{1}{\beta\left(r, N_{r, n_{2}}-R_{r, n_{2}}\right)} G_{m}^{s-1}(u)\left[1-G_{m}(u)\right]^{N_{s, n_{1}}-R_{s, n_{1}}-1}(u) G_{m}^{\prime}(u) \psi_{\alpha, \beta}(u),
$$

where,

$$
\begin{gathered}
\psi_{\alpha, \beta}(u)=G_{m}^{\alpha}(u)\left[1-G_{m}(u)\right]^{\beta}-\lambda, \quad \alpha=r-s>0, \\
\beta=(m+1)^{-1}\left[\left(\bar{m}_{r, n_{2}}+1\right)\left(n_{2}-r\right)-\left(\bar{m}_{s, n_{1}}+1\right)\left(n_{1}-s\right)\right]>0,
\end{gathered}
$$

and $\lambda=\frac{\beta\left(r, N_{r, n_{2}}-R_{r, n_{2}}\right)}{\beta\left(s, N_{s, n_{1}}-R_{s, n_{1}}\right)}$. Since $m>-1, G_{m}(u)=1-(1-u)^{m+1}$ and consequently,

$$
\psi_{\alpha, \beta}(u)=\left[1-(1-u)^{m+1}\right]^{\alpha}(1-u)^{(m+1) \beta}-\lambda .
$$

Therefore, $\psi_{\alpha, \beta}(0)=\psi_{\alpha, \beta}(1)=-\lambda<0$ and

$$
\psi_{\alpha, \beta}^{\prime}(u)=(m+1)\left[1-(1-u)^{m+1}\right]^{\alpha-1}(1-u)^{(m+1) \beta-1}\left[(\alpha+\beta)(1-u)^{m+1}-\beta\right] .
$$

Now, it is necessary to study the sign of $\psi_{\alpha, \beta}^{\prime}(u)$ in the interval $(0,1)$. Clearly, $\psi_{\alpha, \beta}^{\prime}(u)$ and $\left[(\alpha+\beta)(1-u)^{m+1}-\beta\right]$, have the same sign. The following equation,

$$
(\alpha+\beta)(1-u)^{m+1}-\beta=0,
$$

has the only real root $u_{0}=1-\left(\frac{\beta}{\alpha+\beta}\right)^{\frac{1}{m+1}}$ in the interval $(0,1)$. Thus $\psi_{\alpha, \beta}^{\prime}(u)>0$, for $0<u<u_{0}$ and $\psi_{\alpha, \beta}^{\prime}(u)<0$, for $u_{0}<u<1$. Thereupon, the function $\psi_{\alpha, \beta}(u)$ increases if $0<u<u_{0}$ and then decreases when $u_{0}<u<1$. Since $\psi_{\alpha, \beta}(0)=\psi_{\alpha, \beta}(1)=-\lambda<0$, it has exactly two real roots in $(0,1)$. Note that the function $D^{\prime}(u)$ and $\psi_{\alpha, \beta}(u)$ have the same sign. Thereby, the function $\psi_{\alpha, \beta}$ starts with $\psi_{\alpha, \beta}(0)=-\lambda$, increases to intersect the horizontal axis, attains its maximum at $u=u_{0}$ and then decreases to $\psi_{\alpha, \beta}(1)=-\lambda$. In other words, the function $D(x)$ starts from $D(0)=0$, decreases until it takes its minimum and then increases to reach out for its maximum before it decreases again to $D(1)=0$. That is the equation $D(u)=0$ has exactly one real root in the interval $(0,1)$. Therefore, the function $F$ has one growth point beside its right end-point, which complete the proof.

\section{Characterization of Distribution Based on dgos}

Precisely, descending ordered rv's such as lower record values cannot be included in the gos model. Burkschat, et al [17] have introduced dgos as a unified model of descending ordered random variables like reversed order statistics, lower $k$-records and lower Pfeirfer' records, through a combined approach. By analogy with (1.1), the $\operatorname{dgos}, Y_{d}\left(r, n, \tilde{m}_{n}, k\right), r=1,2, \ldots, n$, have been defined in 17, as

$$
Y_{d}\left(r, n, \tilde{m}_{n}, k\right) \stackrel{d}{=} F^{-1}\left(\prod_{j=1}^{r} B_{j}\right) \stackrel{d}{=} F^{-1}\left(\prod_{j=1}^{r} U^{\frac{1}{\gamma_{j}\left(n, \tilde{m}_{n}, k\right)}}\right), r=1,2, \ldots, n .
$$

Hence, $Y_{d}\left(1, n, \tilde{m}_{n}, k\right) \geqslant Y_{d}\left(2, n, \tilde{m}_{n}, k\right) \geqslant \cdots \geqslant Y_{d}\left(n, n, \tilde{m}_{n}, k\right)$ holds almost surely. More details for dual gos can be found in 18$]$ and 19 , among others.

Assume that $Y_{d}\left(i, n_{1}, \tilde{m}_{n_{1}}, k\right), i=1,2, \ldots, n_{1}, Y_{d}\left(j, n_{2}, \tilde{m}_{n_{2}}, k\right), j=1,2, \ldots, n_{2}$ are dgos based on a df $F$, from which $Y_{d}\left(r, n_{2}, \tilde{m}_{n_{2}}, k\right) \stackrel{d}{=} Y_{d}\left(s, n_{1}, \tilde{m}_{n_{1}}, k\right)$, or equivalently,

$$
F_{Y_{d}\left(r, n_{2}, \tilde{m}_{n_{2}}, k\right)}(y)=F_{Y_{d}\left(s, n_{1}, \tilde{m}_{n_{1}}, k\right)}(y), \quad \forall y .
$$

What are conclusions that can be gained about $F$ ? The answer of this question is presented in Theorems 3.1 and 3.2 . The ordered relations presented in Lemma 3.1 is necessary for proving Theorem 3.1, while Lemma 3.2 will be used in the proof of Theorem 3.2 . 
Lemma 3.1. Let $n, r, t$ be positive integers and let $m_{j} \geqslant-1$ be real numbers for all $j=1,2, \ldots, n+t-1$, such that $m_{r+1} \geqslant m_{r+2} \geqslant \cdots \geqslant m_{n+t-1}$. Then,

$$
P\left(Y_{d}\left(r, n, \tilde{m}_{n}, k\right) \geqslant Y_{d}\left(r+t, n+t, \tilde{m}_{n+t}, k\right)\right)=1 .
$$

Theorem 3.1. Let $Y_{d}\left(i, n_{1}, \tilde{m}_{n_{1}}, k\right), i=1,2, \ldots, n_{1}, Y_{d}\left(j, n_{2}, \tilde{m}_{n_{2}}, k\right), j=1,2, \ldots, n_{2}$ be dgos based on the same df $F$, where $\left(n_{1}, n_{2}, r, s\right) \in \mathcal{L}^{C}$. If condition (2.2) and relation (3.2) are satisfied, then $F$ is degenerate $d f$.

Lemma 3.2. Under the same conditions of Lemma 2.4 the marginal df of the $r^{\text {th }}$ dgos is given by

$$
\begin{aligned}
F_{Y_{d}\left(r, n, \tilde{m}_{m}, k\right)}(y) & =I_{F^{m+1}(x)}\left(N_{r, n}-R_{r, n}, r\right) \\
& =\sum_{j=0}^{r-1} \frac{\Gamma\left(N_{r, n}-r m_{r, n}^{*}\right)}{\Gamma(j+1) \Gamma\left(N_{r, n}-r m_{r, n}^{*}-j\right)} F^{(m+1)\left(N_{r, n}-r m_{r, n}^{*}-j-1\right)}(y)\left[1-F^{m+1}(y)\right]^{j},
\end{aligned}
$$

and for $m-$ dgos we have

$$
F_{Y_{d}(r, n, m, k)}(y)=\sum_{j=0}^{r-1} \frac{\Gamma(N)}{\Gamma(j+1) \Gamma(N-j)} F^{(m+1)(N-j-1)}(y)\left[1-F^{m+1}(y)\right]^{j} .
$$

Theorem 3.2. Assume that $\left(n_{1}, n_{2}, r, s\right) \in \mathcal{L}$ and $m_{1}, \ldots, m_{n_{2}-1}$ are real numbers such that $m_{1}=m_{2}=\ldots=m_{r-1}=m$ and $\sum_{j=n_{1}}^{n_{2}-1} m_{j}>(r-s) m$, with $m_{j}>-1$. If $Y_{d}\left(r, n_{2}, \tilde{m}_{n_{2}}, k\right) \stackrel{d}{=} Y_{d}\left(s, n_{1}, \tilde{m}_{n_{1}}, k\right)$, then $F$ has exactly two growth points.

The proofs are similar to the proofs of the corresponding results in Section 2 with suitable modifications.

\section{Remark}

1. The equality (2.1) holds only for discrete distributions.

2. Indeed, all previous results are satisfied for $m$-gos and $m$-dgos models.

3. The representations 2.11) and (3.3) can be used to prove Theorem 2.1 for $m$-gos and Theorem 3.1 for $m$-dgos, respectively.

4. All the previous results remain valid if I replace the set $\mathcal{L}$ by the set

$$
\mathcal{L}^{*}=\left\{\left(n_{1}, n_{2}, r, s\right): n_{1}-n_{2}>s-r>0\right\} .
$$

\section{Acknowledgments}

The author is very grateful to the anonymous reviewers for the valuable comments and suggestions which have improved the manuscript.

\section{References}

[1] U., Kamps, A Concept of Generalized Order Statistics, Teubner, Stuttgart. 1995.

[2] E., Cramer, U. Kamps, Marginal distributions of sequential and generalized order statistics, Metrika 58(2003) 293-310.

[3] U., Kamps, E., Cramer, On distribution of generalized order statistics. Statistics 35(2001) 269-280.

[4] A. E., Aly. Prediction and reconstruction of future and missing unobservable modified Weibull lifetime based on generalized order statistics. J. of Egyptian Math. Soc. 24(2016)(2), 309-318.

[5] H. M., Barakat, E. M., Nigm, M. E. El-Adll, M., Yusuf. Prediction of future generalized order statistics based on exponential distribution with random sample size, Stat. Papers, 59(2018)(2), 605-631.

[6] J., Galambos, S., Kotz, Characterizations of Probability Distributions, Springer, Berlin Heidelberg New York, 1978. 
[7] C. R., Rao, D. N., Chanabhng, Recent approaches to characterizations based on order statistics and record values. In: BRI, (1998) 231-256.

[8] H., Nagaraja, Springer Handbook of Engineering Statistics. Springer-Verlag London, 2006.

[9] U., Gather, U., Kamps, N., Schweitzer, Characterizations of distributions via identically distributed functions of order statistics. In: OrderStatistics: Theory and Methods, Handbook of Statistics, Vol.16, ed. by N. Balakrishnan, C.R. Rao, North-Holland, Amsterdam (1998) 257 - 290.

[10] M., Ahsanullah, A characterization of the uniform distribution by dual generalized order statistics. Comm. Statist. Theory-Methods 33(2004) 2921 - 2928.

[11] I. A., Shah, A. H., Khan, H. M., Barakat, Random translation, dilation and contraction of order statistics. Statist. Probab. Lett. 92(2014) 209-214.

[12] I. A., Shah, A. H., Khan, H. M., Barakat, Translation, contraction and dilation of dual generalized order statistics. Statist. Probab. Lett. 107(2015) 131-135.

[13] V. K., Sagatelyan, Characterization of distributions by equalities of order statistics, j. math. sci. 147(4)(2007) 6946-6949.

[14] J. R., Serfling, Approximation Theorems of Mathematical Statistics, John Wiley \& Sons, Inc., New York, 1980.

[15] H. A., David, H. N., Nagaraja, Order Statistics, Wiley Series in Probability and Statistics, 2003.

[16] H. M., Barakat, Limit theory of generalized order statistics. J. Stat. Plan. Inference. 137(2007) 1-11.

[17] M., Burkschat, E., Cramer, U. Kamps, U., Dual generalized order statistics. Metron 61 (1)(2003) $13-26$.

[18] H. M., Barakat, M. E. El-Adll, M. E. Asymptotic theory of extreme dual generalized order statistics. Statist. Probab. Lett. 79(2009), 1252-1259.

[19] A. A., Ahmed, M. E., El-Adll, T. A., ALOafi. Estimation under Burr type X distribution based on doubly type II censored sample of dual generalized order statistics. J. of Egyptian Math. Soc. 23(2015), 391-396. 